\title{
Needleless Electrospinning of Uniform Nanofibers Using Spiral Coil Spinnerets
}

\author{
Xin Wang, ${ }^{1}$ Haitao Niu, ${ }^{2}$ Xungai Wang, ${ }^{2}$ and Tong Lin ${ }^{2}$ \\ ${ }^{1}$ School of Textile Science and Engineering, Wuhan Textile University, Wuhan 430073, China \\ ${ }^{2}$ Australian Future Fibres Research and Innovation Centre, Deakin University, Geelong, VIC 3217, Australia
}

Correspondence should be addressed to Tong Lin, tong.lin@deakin.edu.au

Received 21 October 2011; Accepted 9 January 2012

Academic Editor: Gajanan S. Bhat

Copyright ( 2012 Xin Wang et al. This is an open access article distributed under the Creative Commons Attribution License, which permits unrestricted use, distribution, and reproduction in any medium, provided the original work is properly cited.

\begin{abstract}
Polyvinyl alcohol nanofibers were prepared by a needleless electrospinning technique using a rotating spiral wire coil as spinneret. The influences of coil dimension (e.g., coil length, coil diameter, spiral distance, and wire diameter) and operating parameters (e.g., applied voltage and spinning distance) on electrospinning process, nanofiber diameter, and fiber productivity were examined. It was found that the coil dimension had a considerable influence on the nanofiber production rate, but minor effect on the fiber diameter. The fiber production rate increased with the increased coil length or coil diameter, or the reduced spiral distance or wire diameter. Higher applied voltage or shorter collecting distance also improved the fiber production rate but had little influence on the fiber diameter. Compared with the conventional needle electrospinning, the coil electrospinning produced finer fibers with a narrower diameter distribution. A finite element method was used to analyze the electric field on the coil surface and in electrospinning zone. It was revealed that the high electric field intensity was concentrated on the coil surface, and the intensity was highly dependent on the coil dimension, which can be used to explain the electrospinning performances of coils. In addition, PAN nanofibers were prepared using the same needleless electrospinning technique to verify the improvement in productivity.
\end{abstract}

\section{Introduction}

Electrospinning is a simple but effective method to produce polymer nanofibers [1], and electrospun nanofibers have shown enormous application potential in diverse areas $[2,3]$. For a conventional electrospinning setup, a needle-like nozzle is often used. Despite its ability to produce bicomponent nanofibers, the conventional needle electrospinning has a small fiber production rate, typically less than $0.3 \mathrm{~g} / \mathrm{hr}$ per needle, which prevents its practical uses.

Efforts to improve the electrospinning productivity have been made based on different principles, such as increasing the needle number (also called multineedle setup), using airjacket to improve the solution flow rate, and electrospinning from open solution surface (also referred to as "needleless electrospinning"). Multi-needle electrospinning is a straightforward strategy to increase the electrospinning productivity. However, the multi-needle setup usually requires a large operating space, and the relative locations of needles have to be optimized to avoid the strong charge-repulsion between the adjacent solution jets, otherwise unevenly deposited nanofiber mat may be obtained. A regular cleaning device has to be applied to each needle to prevent the blockage of the nozzles during electrospinning, which makes the whole setup inapplicable when thousands of needles are used for the nanofiber production. Dosunmu et al. [4] reported the formation of multiple jets from a cylindrical tube. Later on Varabhas et al. [5] modified this setup to control the ejecting locations of jets. Both setups used fluidic channels to convey the solution for electrospinning, which can still be classified as improved needle electrospinning techniques.

Recently needleless electrospinning setups have been reported to increase nanofiber production rate [4-8]. Instead of being generated from a needle tip or small opening, jets in needleless electrospinning are formed from a widely open liquid surface. Needleless electrospinning appeared as early as 1970s, when Simm et al. [9] filed a patent on using rings to electrostatically spin fibers for filtration applications. Jirsak et al. [7] patented their needleless electrospinning design using a roller or cylinder as the fiber generator (also referred to as 
"spinneret" in this paper), which has been commercialized by Elmarco with the brand name Nanospider. Niu et al. [10] used a disc as the spinneret to produce nanofibers, and they also compared the disc with a cylinder spinneret. Recently, a rotary cone was also used as the fiber generator to electrospin nanofibers needlelessly [11]. Yarin and Zussman [6] used a magnetic fluid underneath the polymer solution to initiate needleless electrospinning. Liu et al. [12] blew air to generate bubbles to assist in electrospinning of nanofibers from a liquid surface.

Theoretically, Lukas et al. [8] demonstrated that the generation of multiple jets from an open liquid surface came from electrically amplified liquid waves. For a onedimensional approximation of the fluid surface, the wavelength $\lambda$, which determines the distance between neighboring jets, can be expressed as follows:

$$
\lambda=\frac{12 \pi \gamma}{2 \varepsilon E_{0}^{2}+\sqrt{\left(2 \varepsilon E_{0}^{2}\right)^{2}-12 \gamma \rho g}},
$$

where $E_{0}$ is the external field strength, $\gamma$ is the surface tension of the liquid, $\rho$ is the liquid density, and $g$ is the gravitational acceleration. It clearly shows that the jet formation in needleless electrospinning is highly influenced by the external electric field strength.

For a three-dimensional setup, the geometry of the spinneret greatly influences the distribution of the electric field intensity thus affecting the electrospinning process and fiber properties. However, it has been difficult to directly measure the electric field intensity of an electrospinning setup due to the high-voltage involved. Finite element method (FEM) is a numerical technique for finding approximate solutions of partial differential equations (PDE), which is used to solve a wide range of physical and engineering PDE problems. It provides an attractive method to analyze the electric field in electrospinning. Since the practical dimensions and material properties can be used for the FEM calculation, it enables one to visualize the electric field intensity profile and to understand how this profile may be influenced by the spinneret geometry as well as material characteristics.

In the previous work, we have used disc and cylinder as spinnerets to electrospin polyvinyl alcohol (PVA) nanofibers, and demonstrated the noticeable differences between disc and cylinder electrospinning processes [10]. The disc produced finer nanofibers with a narrower diameter distribution compared to the cylinder. A disc spinneret $2 \mathrm{~mm}$ thick has roughly the same fiber production rate as a cylinder of the same diameter $(80 \mathrm{~mm})$ but 100-times larger in length (i.e., $20 \mathrm{~cm}$ ). Using FEM, we have also analyzed the electric field in the needleless electrospinning setup, and confirmed that these differences came from different electric field distributions. Compared with the conventional needle electrospinning, both cylinder and disc electrospinning produced coarser nanofibers.

In parallel, we also used a conical wire coil to electrospin PVA nanofibers [13]. It was interesting to find that the conical coil setup produced finer nanofibers with a higher productivity compared to the conventional needle electrospinning. However, it was difficult to scale up the conical coil system, which hampered the effort to further improve the productivity. In our recent work, a spiral wire coil was used as the spinneret to electrospin nanofibers. We found that the spiral coil produced finer nanofibers with a narrower diameter distribution than the needle electrospinning. A finite element method was used to understand the electric field intensity profile around the coil surface and the influences of coil dimension on the electric field intensity profile and electrospinning performance.

\section{Experimental}

2.1. Materials and Measurements. PVA (average molecular weight 146,000-186,000, 96\% hydrolyzed), polyacrylonitrile (PAN, average molecular weight 70,000), and dimethylformamide (DMF) were obtained from Aldrich-Sigma. The fiber morphology was observed under scanning electron microscope (SEM, Leica S440). The average fiber diameter was calculated from the SEM images using an image analysis software (ImagePro+6.0). More than 100 fibers were counted from at least 4 SEM images which were taken from different places of a sample. The productivity of needle electrospinning was calculated based on the largest flow rate of the polymer solution being processed while no liquid dropped from the needle tip. For needleless electrospinning, the productivity was measured based on the dry weight of collected nanofibers. The crystalline structure of electrospun nanofibers was tested using Panalytical XRD.

2.2. Needleless Electrospinning. The apparatus used for needleless electrospinning of nanofibers is depicted in Figure 1(a). A spiral coil made from a copper wire was used as the fiber generator. The polymer solution was loaded onto the coil surface by the slow rotation of the coil. The rotating speed was controlled at $40 \mathrm{rpm}$. The solution was charged with a high-voltage power supply (ES100P, Gamma High Voltage Research) through inserting an electrode into the solution. A rotating drum covered with aluminum foil was used to collect nanofibers. The applied voltage, collecting distance, and PVA concentration were $60 \mathrm{kV}, 13 \mathrm{~cm}$, and $9 \mathrm{wt} \%$, respectively, unless otherwise stated. Unless otherwise specified, the coil length, diameter, spiral distance, and wire diameter were set at $16 \mathrm{~cm}, 8 \mathrm{~cm}, 4 \mathrm{~cm}$ in, and $2 \mathrm{~mm}$, respectively.

2.3. Electric Field Analysis. The electric field was calculated using a finite element method (FEM) program package COMSOL3.5. Before the calculation, the physical geometries of the electrospinning setups (e.g., spinneret, solution container, and collector), polymer solution in the container, and collector were established according to their practical dimensions, locations, and relative permittivities. A high voltage was then set to the metal wire located at the bottom of the solution bath. The metal collector and the boundaries at an infinite distance were set as zero potential. All the other boundaries were set as continuity. The meshing and solving were performed by the software to obtain the electric field intensity and profile. 


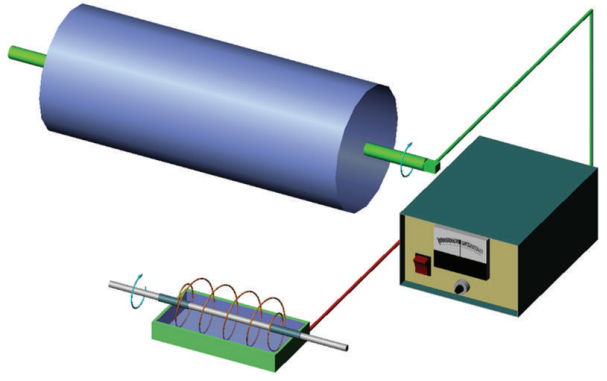

(a)

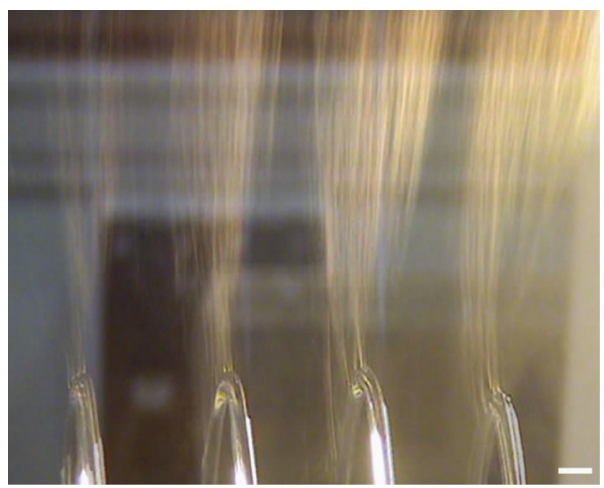

(c)

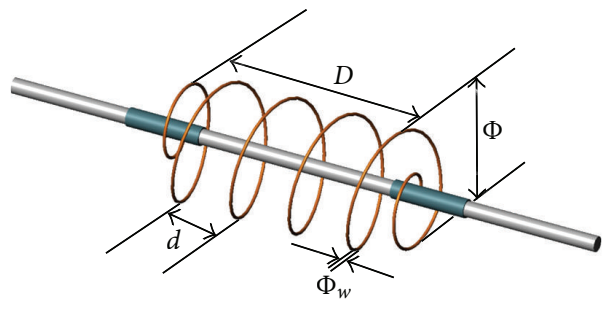

(b)

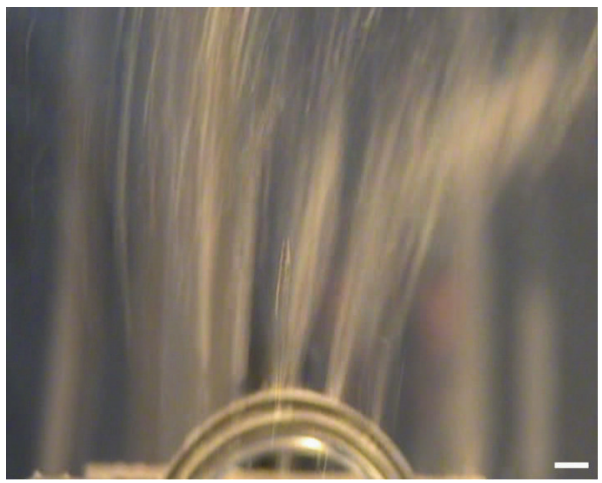

(d)

FIGURE 1: (a) Schematics of spiral coil electrospinning setup; (b) magnified view of the coil; photos of spiral coil spinning processes. (c) front view and $(\mathrm{d})$ side view. bar $=1 \mathrm{~cm}$.

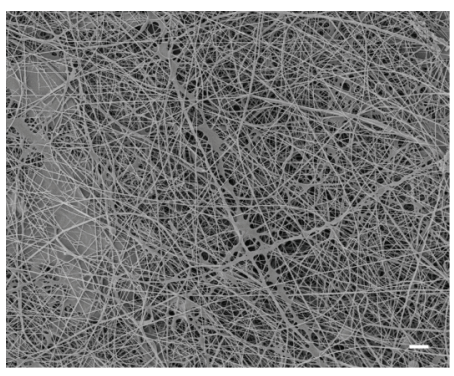

(a)

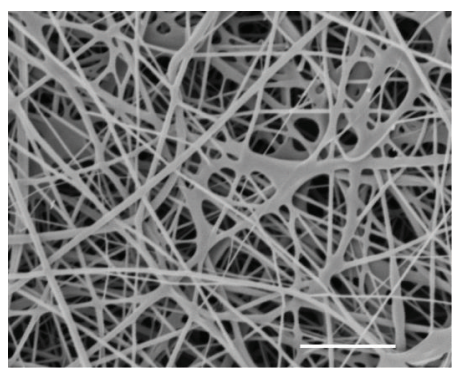

(b)

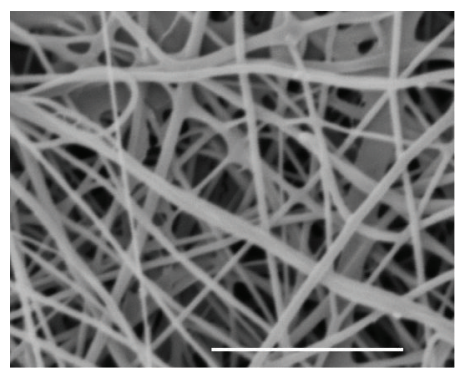

(c)

FIGURE 2: SEM images of PVA nanofibers from spiral wire coil electrospinning (magnification is $1 \mathrm{k}, 5 \mathrm{k}$, and $10 \mathrm{k}$, resp., bar $=5 \mu \mathrm{m}$ ).

\section{Results and Discussion}

Figure 1(a) illustrates the coil electrospinning setup. A spiral coil made from a metal wire was used as the fiber generator, which was partly immersed in the polymer solution. The polymer solution was loaded on to the coil surface because of the slow rotation of the coil. The viscoelastic property of the polymer solution also assisted in the formation of an evenly distributed solution layer on each spiral. When the coil together with the polymer solution was charged with an electric voltage higher than $40 \mathrm{kV}$, a number of jets were generated from the coil surface. With the increased applied voltage, more jets were formed. Figures $1(\mathrm{c})$ and $1(\mathrm{~d})$ show the front and side views of coil electrospinning process. A large number of polymer jets were clearly observed to be produced from each coil section. The side view shows that the polymer jets were produced from the top of a circular sector zone with a central angle of about 90 degrees (Figure 1(d)). A voltage up to $70 \mathrm{kV}$ could be applied for electrospinning, and a further increase in the voltage led to "corona discharges" instead of normal electrospinning.

Figure 2 shows the morphology of electrospun PVA nanofibers. Some fibers were observed to stick to each other forming an interconnected fibrous structure. The formation of bonded fibrous structure was because of the insufficient solvent (water) evaporation from the polymer jets. This usually happened to PVA, especially when lots of jets were generated simultaneously in a very limited space during needleless electrospinning.

Figures 3(a)-3(d) shows the SEM images of PVA fibers electrospun from PVA solutions with different concentrations. Beaded-fibers appeared when the PVA concentration 


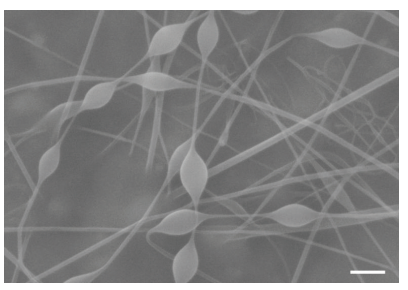

(a)

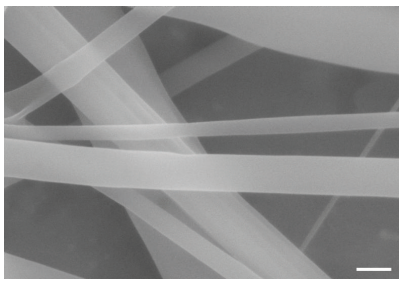

(c)

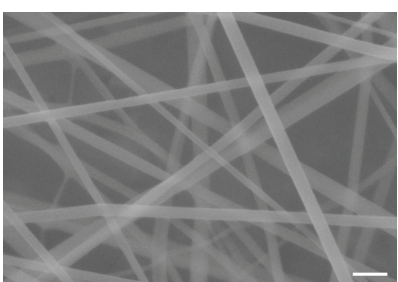

(b)

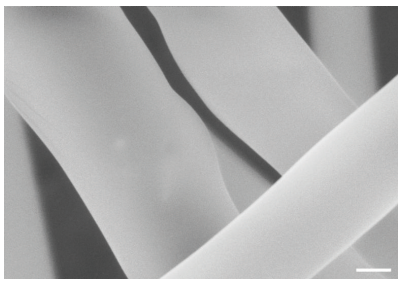

(d)

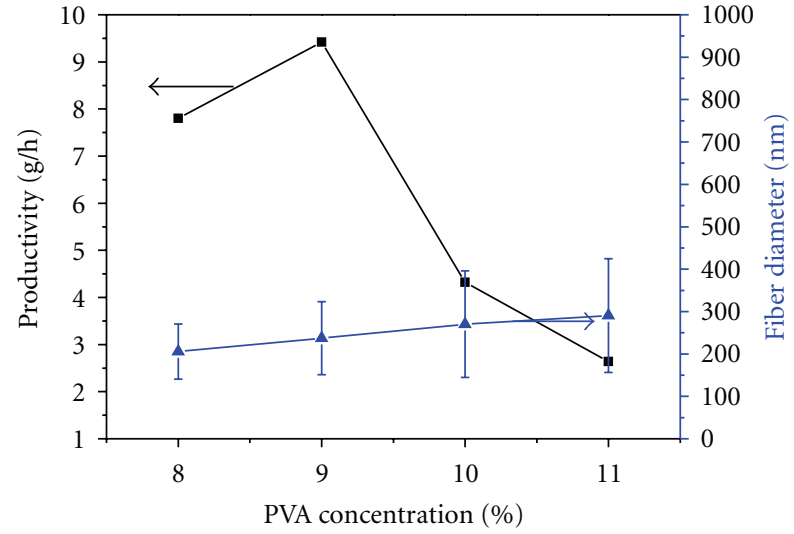

-- Productivity

-A- Fibre diameter

(e)

FIgURE 3: SEM images of PVA nanofibers from spiral wire coil electrospinning with different PVA concentrations. (a) 6\%, (b) 9\%, (c) 12\%, and (d) $15 \%$ (bar $=1 \mu \mathrm{m}$ ). (e) Effects of PVA concentration on the fiber diameter and productivity.

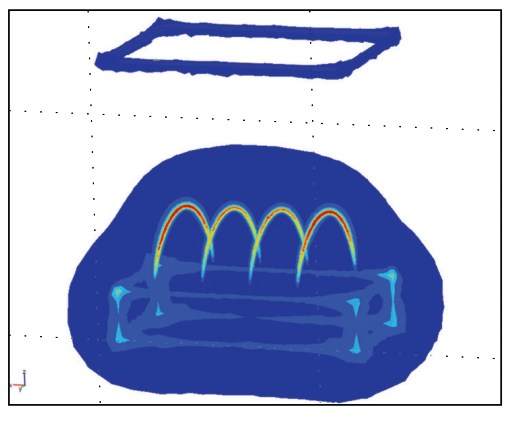

(a)

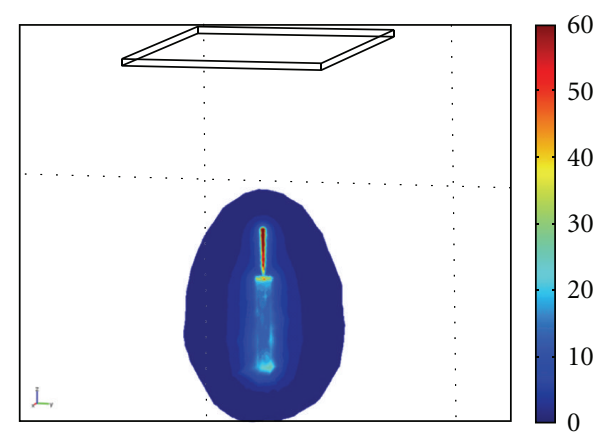

(b)

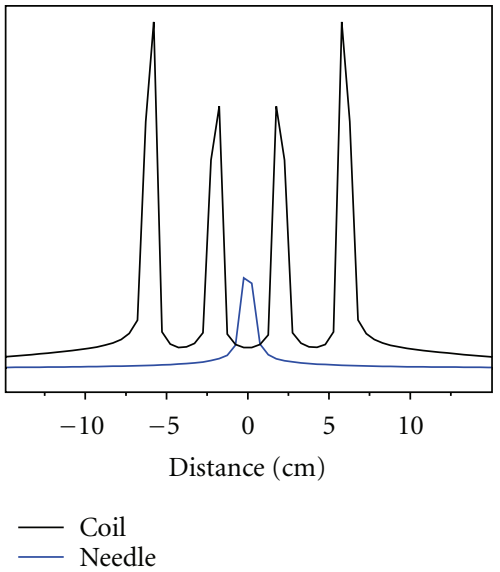

(d)

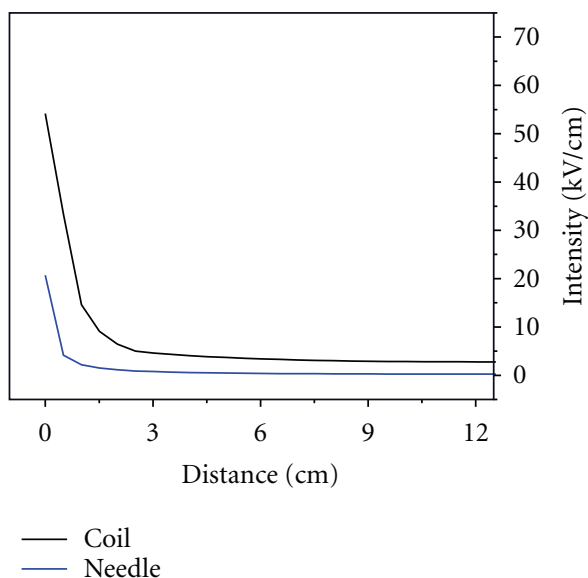

(e)

FIGURE 4: Electric field intensity profiles of (a) spiral wire coil electrospinning, (b) needle electrospinning, and (c) schematic illustration of a setup used to verify the influence of protrusion length on electric field intensity. (d) Electric field intensities along the coil axis direction and (e) from fiber generators to the collector. 


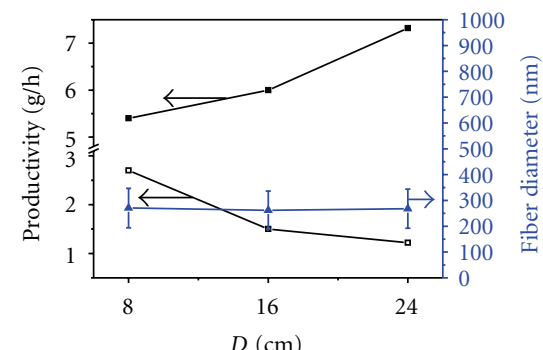

-^- Fibre diameter

--- Productivity

$-\square-$ Productivity of each spiral

(a)

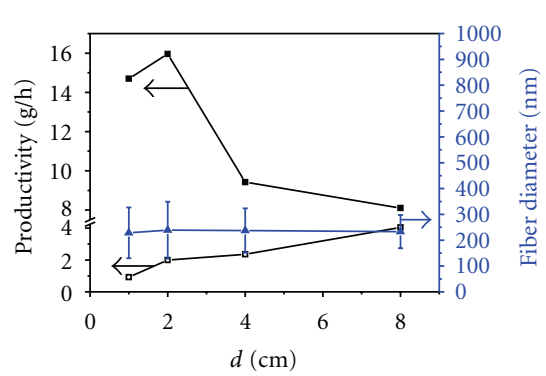

- - Productivity

- - Productivity of each spiral

-₫-Fibre diameter

(b)

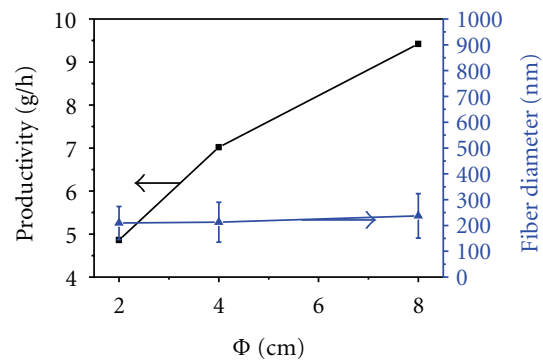

- - Productivity

-\$- Fibre diameter

(c)

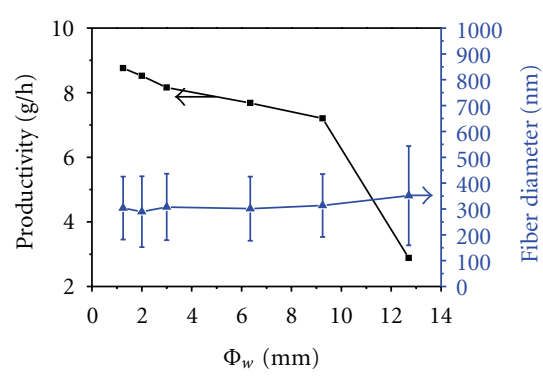

- Productivity

-^- Fiber diameter

(d)

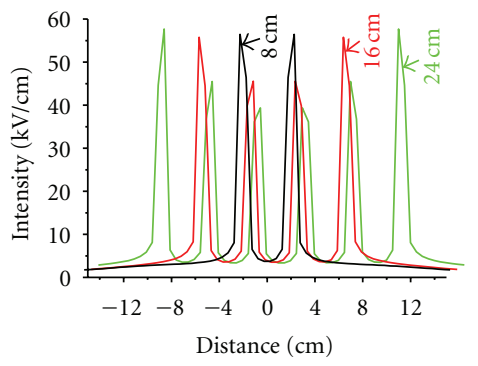

(a')

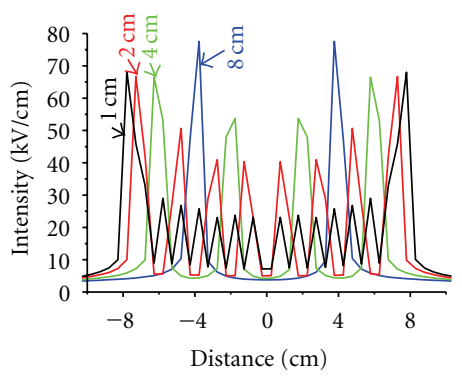

(b')

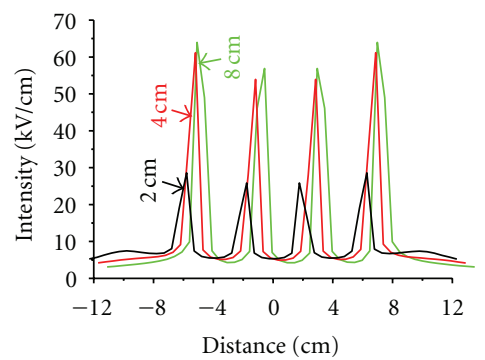

(c')

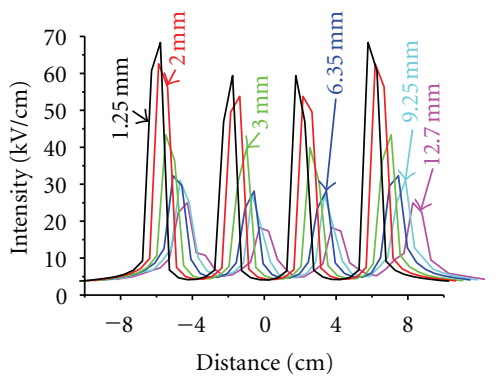

(d')

Figure 5: Effects of coil dimensions on the fiber diameter, productivity, and electric field intensity, (a) and (a') coil length D; (b) and (b') spiral distance $d$; (c) and (c') coil diameter $\Phi$; (d) and (d') wire diameter $\Phi_{w}$. 


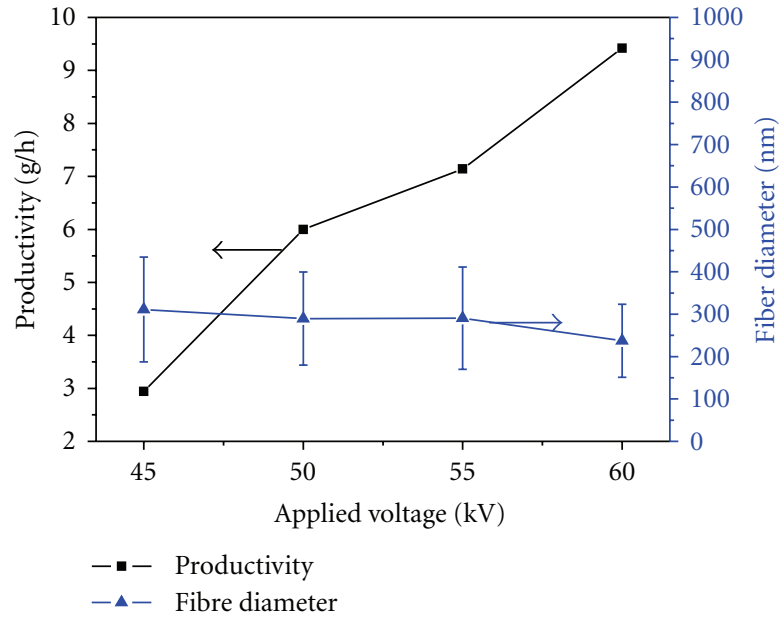

(a)

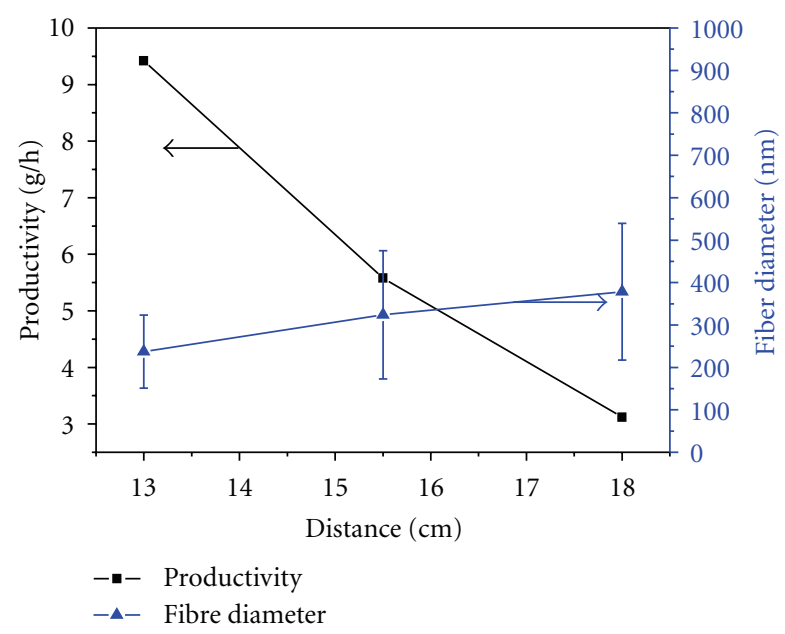

(b)

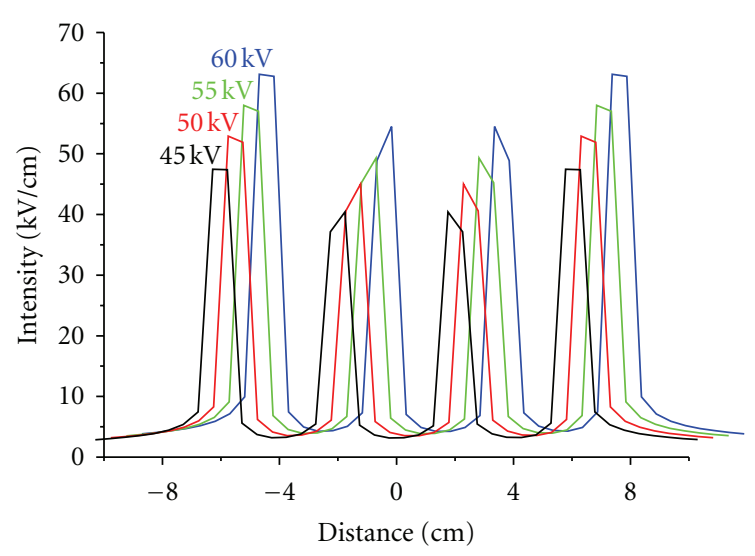

$\left(a^{\prime}\right)$

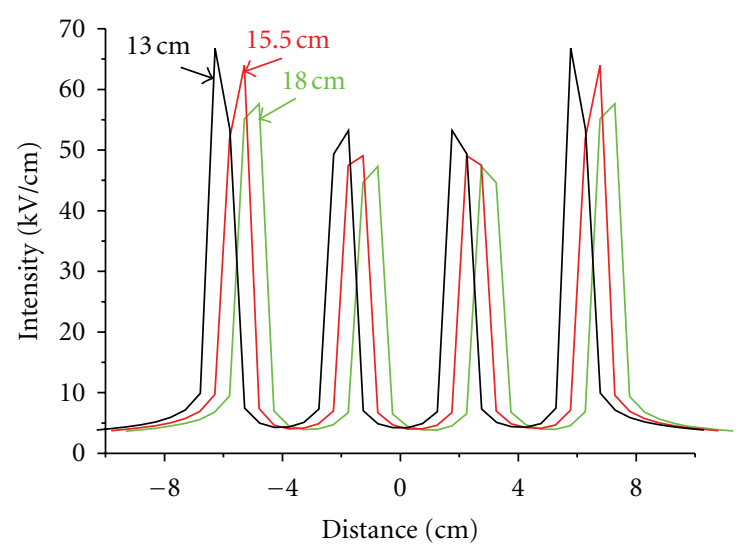

(b')

FIGURE 6: Effects of applied voltage and collecting distance on the fiber diameter and productivity. (a) and (a') applied voltage; (b) and (b') collecting distance.

was lower than $8 \mathrm{wt} \%$. With the increased PVA concentration, the fiber diameter increased greatly (Figures $3(\mathrm{~b})-3(\mathrm{~d})$ ).

The fiber diameter increased with the increase of PVA concentration in the range of $8-11 \mathrm{wt} \%$. The increased polymer concentration also resulted in a reduced number of polymer jets (Figure 3(e)) when the solution concentration was over $9 \mathrm{wt} \%$, which resulted in a lower fiber production rate. When the PVA concentration was higher than $15 \mathrm{wt} \%$, no fibers could be electrospun due to the high solution viscosity. For comparison, the same PVA solutions were also electrospun using a conventional needle electrospinning setup [14]. When the PVA concentration was higher than $12 \mathrm{wt} \%$, clogging of the polymer solution within the needle always happened due to the high solution viscosity. In such a case, a much higher applied voltage had to be used. However, the "corona" discharge happened when the applied voltage was higher than $24 \mathrm{kV}$ in the experiment.

The electric field calculation results indicated that the electric field distributed unevenly along the coil surface, and the coil dimension affected the electric field greatly. As illustrated in Figure 4(a), the coil surface had a high electric field because the coil wire had a small radius. Such an intensified electric field can be found in all coil sections. For comparison, the electric field of a needle setup was also calculated. As shown in Figure 4(b), the high electric field was mainly concentrated on the needle surface and the highest electric field intensity was at the needle tip. Electric field intensities along the coil axis direction and from the fiber generators to the collectors are shown in Figures 4(d) and 4(e), respectively. Under the respective applied voltages, the electric field intensity of the coil setup was higher than that of the needle setup. It was also revealed that the high electric field intensity peaks were generated on the top of the coil whereas the tip of the needle just showed one peak with a lower intensity. Since the electric field is the main driving force to initiate the formation of jets [15], a polymer solution charged by a stronger electric field is easier to generate solution jets, and the jets should be highly stretched under 


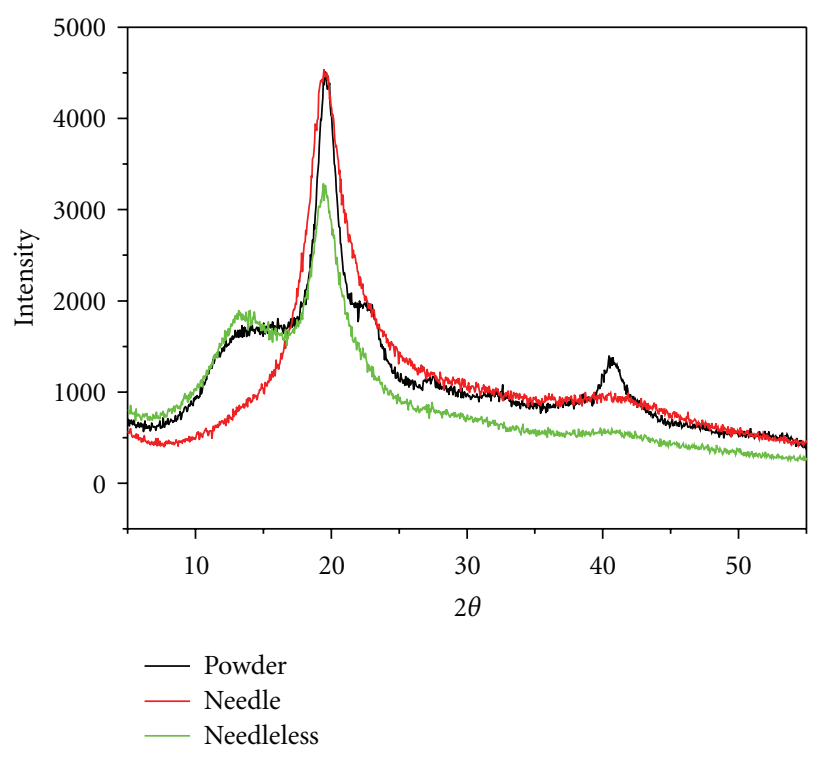

FIGURe 7: WXRD patterns of PVA powder and PVA nanofibers.

the stronger electric force, hence producing finer and more uniform fibers with a much higher production rate.

By combining the electric field analysis with the experimental results, the influences of coil dimension on the fiber diameter and productivity can be understood. The fiber diameter was hardly affected by the coil length $D$. The productivity improved with the increased coil length $D$ (Figure 5(a)). Figure 5(a') shows the electric field intensities of spiral coils with different coil length $D$. Although the electric field intensity in the middle area decreased with the increased $D$, it was still over the intensity threshold for generating nanofibers. Longer coils generated more polymer jets. However, due to the decline of electric field intensity in the middle area (Figure 5(a')), each single spiral showed a reduced fiber production rate in the meantime.

When the spiral distance $d$ (pitch) decreased from $8 \mathrm{~cm}$ to $2 \mathrm{~cm}$, the electrospinning productivity improved and the productivity of each spiral decreased gradually (Figure 5(b)). The spiral distance had little influences on the fiber diameter and diameter distribution. The variation of $d$ affected the electric field intensity of coil greatly (Figure 5(b')). It was found that electric field intensity on the side coil surface was higher than that on the middle coil. When $d$ was larger than $1 \mathrm{~cm}$, the electric field in the coil middle area was strong enough to produce nanofibers, therefore the productivity of coil increased with the reduced $d$. When $d$ was $1 \mathrm{~cm}$, the electric field intensity in the coil middle area was much lower than that from the coil $(d=2 \mathrm{~cm})$ and can only produce nanofibers intermittently, resulting in a slightly reduced productivity (Figure $5(\mathrm{~b})$ ).

Both coil diameter $(\Phi)$ and wire diameter $\left(\Phi_{w}\right)$ had a considerable influence on the nanofiber productivity, but these parameters only had a slight effect on the fiber diameter. The productivity of coil electrospinning increased evidently with the increased coil diameter (Figure 5(c)) and the decreased wire diameter (Figure 5(d)), which was attributed to the stronger electric fields generated by the larger diameter coils (in the range of $2 \mathrm{~cm}-8 \mathrm{~cm}$ ) (Figure 5(c')) and the finer wire coil (Figure 5(d')).

In physics, the charge density on the surface of an irregularly shaped conductor is high in convex regions with a small radius of curvature. In our case, the wire with smaller diameter should have larger electric field intensity because of the smaller radius of curvature. In the case of forming greater intensity of electric field on a coil with larger coil diameter, this was attributed to the long protrusion of large coil and the influence of solution bath. In our design, the high voltage was charged on the solution bath underneath the coil. Because the liquid bath formed second electric field, it influenced the intensity of electric field on the coil surface above. This effect was verified through a simple calculation using a structure shown in Figure 4(c). When the distance between the needle tip and the counter electrode remained the same, with an increase in the needle length (" $x$ " in Figure 4(c)) from $1 \mathrm{~cm}$, to $2 \mathrm{~cm}$ and $4 \mathrm{~cm}$, the electric field intensity on the needle tip (“*” marked in Figure 4(c)) increased from $17.7 \mathrm{kV} / \mathrm{cm}$, to $19.3 \mathrm{kV} / \mathrm{cm}$ and $20.2 \mathrm{kV} / \mathrm{cm}$, respectively.

The increase in electric field strength could lead to the formation of more "Taylor cones" from the coil surface and jets travelled under a higher electric field were also faster. Both effects favored to the increase in the fibre productivity.

The influences of applied voltage and electrospinning distance on the electrospinning process can be well interpreted with help of electric field analysis. For coil electrospinning, the minimum applied voltage for inducing the fiber generation was around $40 \mathrm{kV}$. Higher applied voltage led to more stable electrospinning until "corona discharge" occurred when the applied voltage was higher than a critical value. As shown in Figure 6(a), the productivity increased when a higher voltage was applied, and the increased applied voltage also assisted in narrowing the fiber diameter distribution (Figure 6(a)). This trend was exactly the same with the conventional needle electrospinning because the increased applied voltage improved the electric field strength (Figure 6(a')). Reducing the collecting distance had an opposite effect. The productivity of coil electrospinning reduced with the increased collecting distance (Figure 6(b)), because a larger collecting distance led to the weakened electric field (Figure 6(b')).

The WAXD patterns of PVA powder and PVA nanofibers from both needle and needleless electrospinning are shown in Figure 7. PVA powder showed typical diffraction peaks (100) at $2 \theta=12.4^{\circ},(101)$ at about $2 \theta=19.4^{\circ},(200)$ at $2 \theta=23.0^{\circ}$, and (111) at $2 \theta=40.5^{\circ}$. For nanofibers electrospun from needle electrospinning, just a prominent peak (101) at about $2 \theta=19.4^{\circ}$ and a small peak at $2 \theta=$ $40.5^{\circ}$ were observed. However, nanofibers from needleless electrospinning showed a similar trend to that of PVA powder, except that the intensity of the peak at $2 \theta=19.4^{\circ}$ was lower. The differences are probably due to the differences in water content and humidity level during electrospinning $[16,17]$. The polymer crystalline in electrospun fibers is an important property determining the mechanical strength. The improvement of fiber tensile strength through increasing the polymer crystallinity has been reported [18, 19]. 


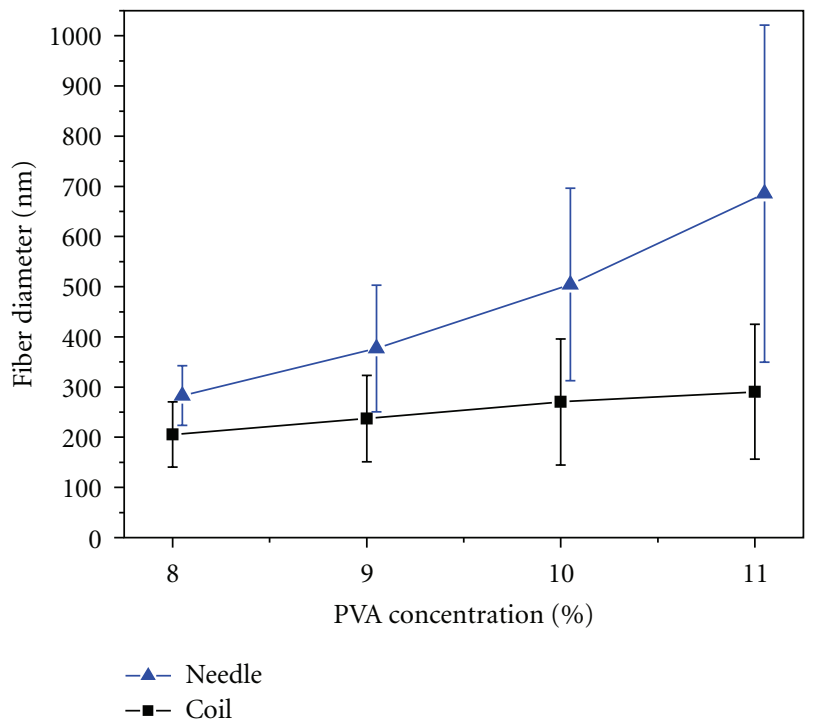

(a)

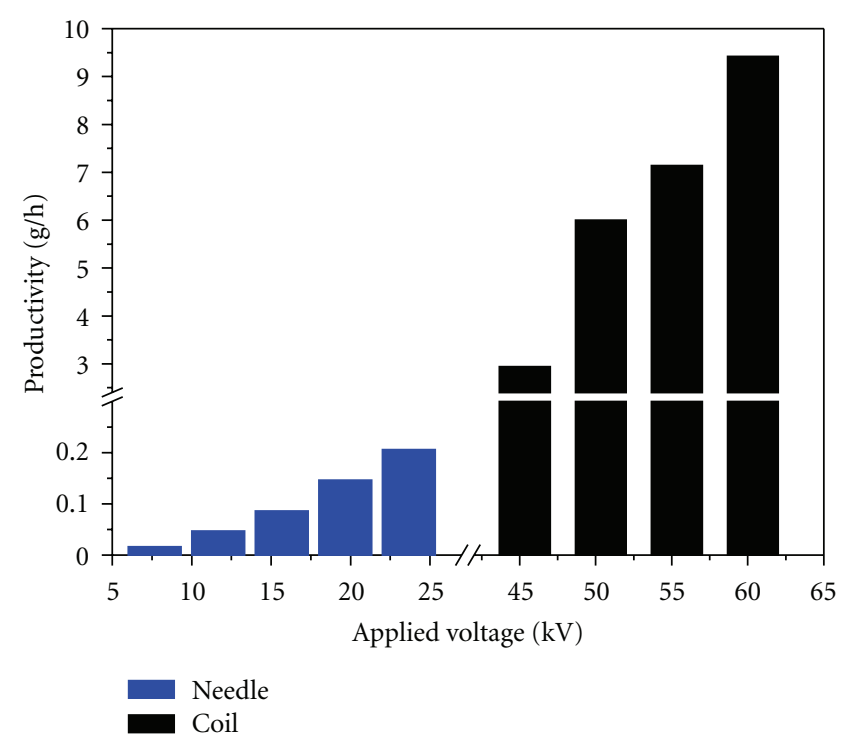

(b)

FIGURE 8: Comparison between needle electrospinning and spiral wire coil electrospinning, (a) fiber diameter, and (b) productivity.

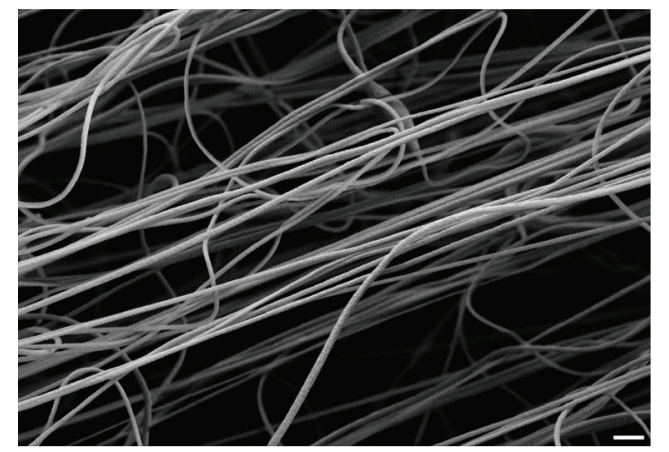

(a)

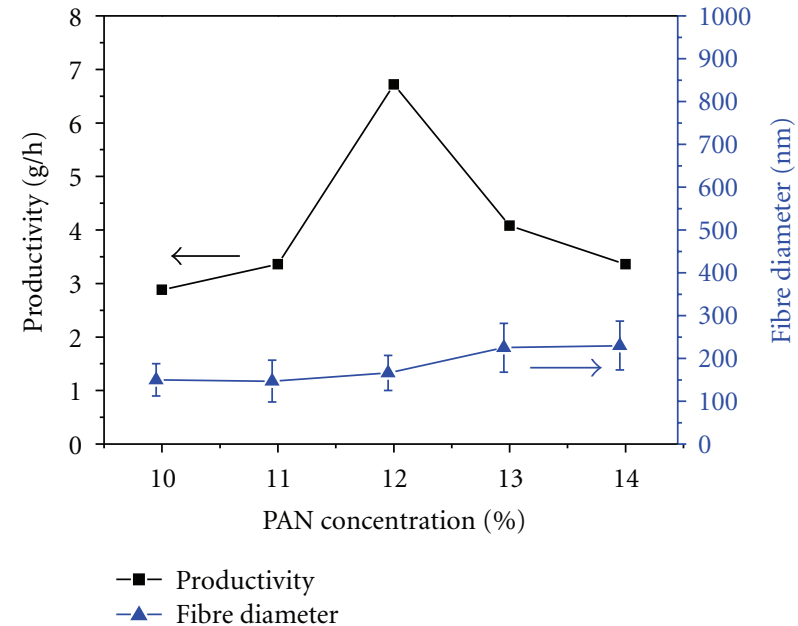

(b)

Figure 9: (a) SEM images of coil electrospun PAN fibers (PAN concentrations $=12 \mathrm{wt} \%$, bar $=1 \mu \mathrm{m}$ ); (b) effects of PAN concentration on the fiber diameter and productivity.

Nanofibers containing higher crystallinity generally have larger tensile strength. The higher crystallinity of needleless electrospun nanofibers suggests that they may have better mechanical strength than needle electrospun nanofibers.

The coil spinneret showed a better electrospinning performance compared with needle electrospinning. In coil electrospinning, the fiber diameter increased from $205.4 \pm$ $65.1 \mathrm{~nm}$ to $290.8 \pm 134.2 \mathrm{~nm}$ when the PVA concentration increased from $8 \%$ to $11 \%$ (Figure $8(a)$ ). For needle electrospinning, the fiber diameter increased from $283.0 \pm 59.4 \mathrm{~nm}$ to $685.6 \pm 336.0 \mathrm{~nm}$. The nanofibers produced from coil spinneret were much thinner with a narrower fiber diameter distribution compared to those from needle electrospinning, especially when the PVA concentration was higher than $9 \%$. Figure 8(b) shows the productivities of needle and needleless electrospinning. The productivity of needle electrospinning was from $0.01 \mathrm{~g} / \mathrm{hr}$ to $0.21 \mathrm{~g} / \mathrm{hr}$ when the applied voltage increased from $8 \mathrm{kV}$ to $24 \mathrm{kV}$. The productivity of coil needleless electrospinning increased from $2.94 \mathrm{~g} / \mathrm{hr}$ to $9.42 \mathrm{~g} / \mathrm{hr}$ when the applied voltage increased from $45 \mathrm{kV}$ to $60 \mathrm{kV}$. The applied voltage played a crucial role in improving the productivity of coil electrospinning.

The above results were based on electrospinning of PVA solutions, other materials, such as PAN, were also electrospun using the same setup and similar trends were obtained. Figure 9(a) shows the fiber morphology of electrospun PAN 
nanofibers, which had a uniform fiber structure. Similar to PVA, the productivity of PAN nanofibers increased with the increased applied voltage. The average fiber diameter increased when the PAN concentration increased from $11 \mathrm{wt} \%$ to $14 \mathrm{wt} \%$ (Figure $9(\mathrm{~b})$ ). The productivity did not improve monotonically with the increased PAN concentration but declined when the PAN concentration was over $12 \mathrm{wt} \%$ (Figure 9(b)). The different spinning performances of PVA and PAN reflect differences in their intrinsic characteristics. The production rate of PAN nanofibers for needle electrospinning was $0.6 \mathrm{~g} / \mathrm{hr}$, which was much lower than that of coil electrospinning.

\section{Conclusions}

We have demonstrated a novel needleless electrospinning setup by using a spiral wire coil as the fiber generator. This device has been used to produce PVA and PAN nanofibers successfully. The PVA nanofiber production rate was much higher than that of single-needle electrospinning. Coil electrospinning also produced finer nanofibers than needle electrospinning. The productivity of coil electrospinning increased with the increased applied voltage and decreased collecting distance. Electric field analysis showed that high electric field intensity was generated around each coil spiral and the intensity was much higher than that of needle electrospinning nozzle. The electric field distribution was an important factor affecting the electrospinning process, fiber production rate, and resultant nanofiber property. Increasing the electric field intensity led to stronger electrostatic force resulting in thinner fibers and higher fiber productivity. This setup shows great potential in the large-scale production of nanofibers which will contribute to not only the laboratory research but also the industrialization of electrospinning.

\section{Authors' Contribution}

The first two authors have equal contribution to the paper. This work was conducted in Deakin university, Austrialia, while the first author was doing his $\mathrm{PhD}$ study.

\section{References}

[1] D. H. Reneker and I. Chun, "Nanometre diameter fibres of polymer, produced by electrospinning," Nanotechnology, vol. 7, no. 3, pp. 216-223, 1996.

[2] A. Greiner and J. H. Wendorff, "Electrospinning: a fascinating method for the preparation of ultrathin fibers," Angewandte Chemie, vol. 46, no. 30, pp. 5670-5703, 2007.

[3] D. Li and Y. Xia, "Electrospinning of nanofibers: reinventing the wheel?" Advanced Materials, vol. 16, no. 14, pp. 1151-1170, 2004.

[4] O. O. Dosunmu, G. G. Chase, W. Kataphinan, and D. H. Reneker, "Electrospinning of polymer nanofibres from multiple jets on a porous tubular surface," Nanotechnology, vol. 17, no. 4, pp. 1123-1127, 2006.

[5] J. S. Varabhas, G. G. Chase, and D. H. Reneker, "Electrospun nanofibers from a porous hollow tube," Polymer, vol. 49, no. 19, pp. 4226-4229, 2008.
[6] A. L. Yarin and E. Zussman, "Upward needleless electrospinning of multiple nanofibers," Polymer, vol. 45, no. 9, pp. 29772980, 2004.

[7] O. Jirsak, F. Sanetrnik, D. Lukas, V. Kotek, L. Martinova, and J. Chaloupek, "A method of nanofibres production from a polymer solution using electrostatic spinning and a device for carrying out the method," WO 2005/024101 A1, 2005.

[8] D. Lukas, A. Sarkar, and P. Pokorny, "Self-organization of jets in electrospinning from free liquid surface: a generalized approach," Journal of Applied Physics, vol. 103, no. 8, Article ID 084309, 2008.

[9] W. Simm, C. Gosling, R. Bonart, and B. Von Falkai, "Fibre fleece of electrostatically spun fibres and methods of making same," US 4143196, 1979.

[10] H. Niu, T. Lin, and X. Wang, "Needleless electrospinning. I. A comparison of cylinder and disk nozzles," Journal of Applied Polymer Science, vol. 114, no. 6, pp. 3524-3530, 2009.

[11] B. Lu, Y. Wang, Y. Liu et al., "Superhigh-throughput needleless electrospinning using a rotary cone as spinneret," Small, vol. 6, no. 15, pp. 1612-1616, 2010.

[12] Y. Liu, J. H. He, and J. Y. Yu, "Bubble-electrospinning: a novel method for making nanofibers," Journal of Physics, vol. 96, no. 1, Article ID 012001, 2008.

[13] X. Wang, H. Niu, T. Lin, and X. Wang, "Needleless electrospinning of nanofibers with a conical wire coil," Polymer Engineering and Science, vol. 49, no. 8, pp. 1582-1586, 2009.

[14] T. Lin, J. Fang, H. Wang, T. Cheng, and X. Wang, "Using chitosan as a thickener for electrospinning dilute PVA solutions to improve fibre uniformity," Nanotechnology, vol. 17, no. 15, article no. 017, pp. 3718-3723, 2006.

[15] J. M. Deitzel, J. Kleinmeyer, D. Harris, and N. C. Beck Tan, "The effect of processing variables on the morphology of electrospun nanofibers and textiles," Polymer, vol. 42, no. 1, pp. 261-272, 2001.

[16] H. E. Assender and A. H. Windle, "Crystallinity in poly(vinyl alcohol). 1. An X-ray diffraction study of atactic PVOH," Polymer, vol. 39, no. 18, pp. 4295-4302, 1998.

[17] M. L. Minus, H. G. Chae, and S. Kumar, "Single wall carbon nanotube templated oriented crystallization of poly(vinyl alcohol)," Polymer, vol. 47, no. 11, pp. 3705-3710, 2006.

[18] X. Zong, S. Ran, D. Fang, B. S. Hsiao, and B. Chu, "Control of structure, morphology and property in electrospun poly(glycolide-co-lactide) non-woven membranes via postdraw treatments," Polymer, vol. 44, no. 17, pp. 4959-4967, 2003.

[19] B. Gupta, N. Revagade, N. Anjum, B. Atthoff, and J. Hilborn, "Preparation of poly(lactic acid) fiber by dry-jet-wetspinning. I. Influence of draw ratio on fiber properties," Journal of Applied Polymer Science, vol. 100, no. 2, pp. 12391246, 2006. 

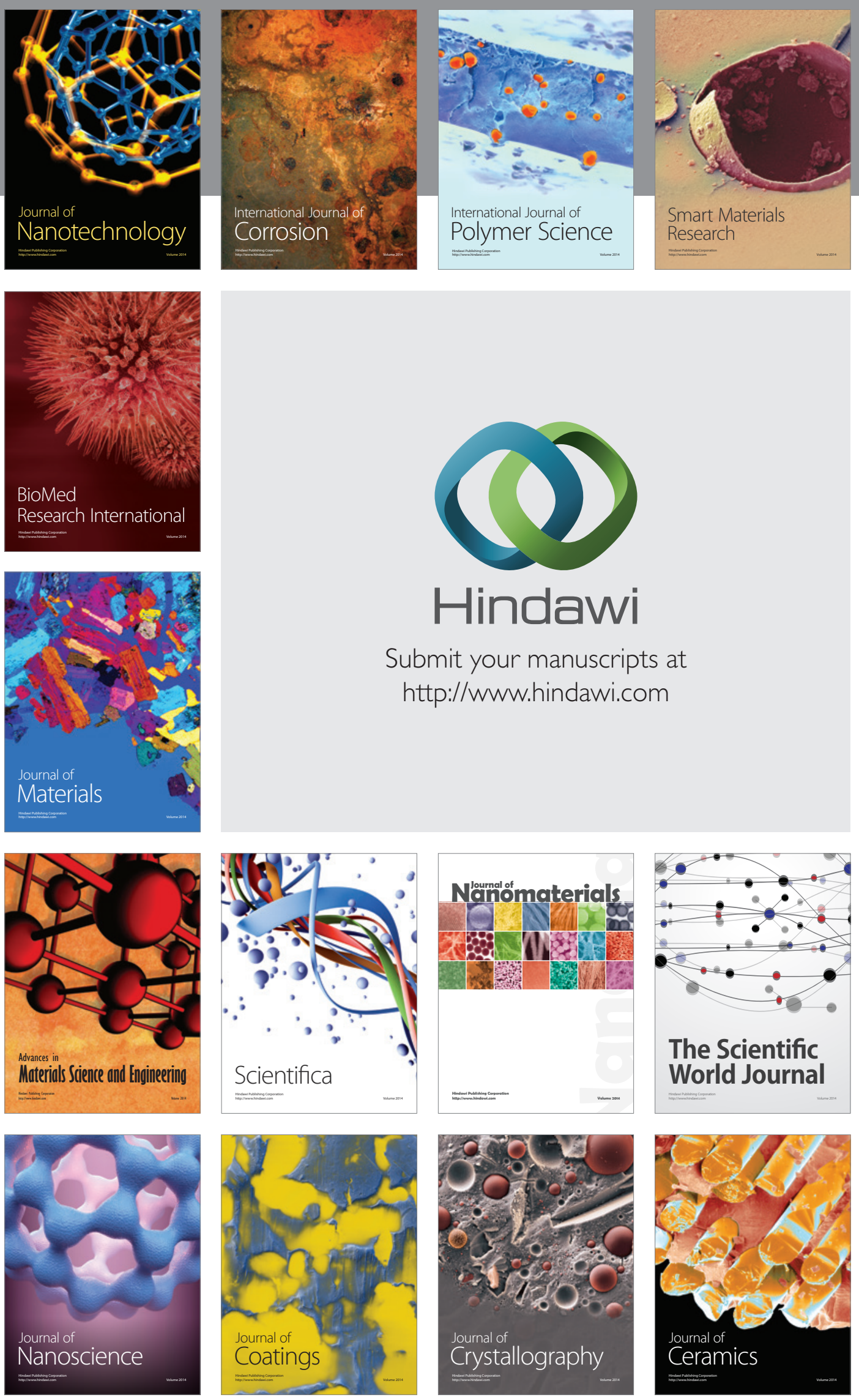

The Scientific World Journal

Submit your manuscripts at

http://www.hindawi.com

\section{World Journal}

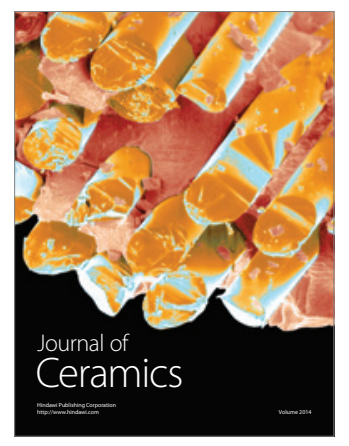

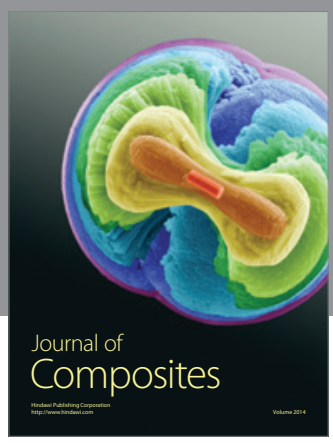
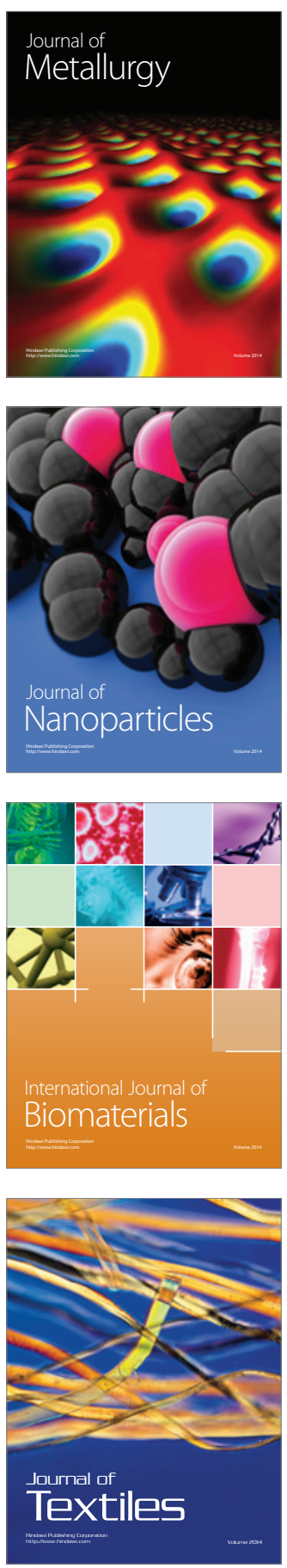